\title{
WS1-2-3
}

\section{A Comparative Study of the Oncological Long-term Outcomeof Laparoscopic Nephroureterectomy and Standard Nephroureterectomy For Upper Urinary Tract Trasitionalcell Carcinoma}

\author{
Department of Urology, Okayama University Graduate School of Medicine, Okayama, Japan \\ Yasuyuki Kobayashi, Takashi Saika, Toyohiko Watanabe, Shinya Uehara, Yasutomo Nasu, Hiromi Kumon
}

INTRODUCTION AND OBJECTIVE:To reveal the oncological safety of laparoscopic nephroureterectomy(LNU),We compared the long-term oncological outcome of laparoscopic versus openNephroureterectomy(ONU) in patients with upper tract traditional cell caricinoma(TCC). METHODS;Three hundred sixty-seven nephroureterectomy were performed at our institutes for upper tract TCC without distant metastases. Fifty-eight LNUs with open intact specimen retrieval n conjunction with open distal ureter and bladder cuff removal,and 165 ONUs of 223 patients without concomitant or prior bladder cancer were reviewed and analyzed retorospectively. Mean follow-up was 25.7(1-58)months for LNU and 36.1 (range 1-79) for ONU. RESULTS.:Bladder recurrence was recognized in 19 patients (32.8\%) following LNU with a median duration of 5.7 month, compared with 69 patients $(41.2 \%)$ after ONU, with LNU. There were distant metastases in 15 patients $(25.9 \%)$ after LNU and in 38 patients $(23.0 \%)$ after ONU. The frequency of bladder recurrence, local recurrence.and distant metastases did not differ siginificantly in both groups the two-year cause specific survival rates were similar $(85.5 \%$ vs $86.7 \%$ ). In all patients the risk of metastases and death were increased by advanced tumor stage and grade,but not by surgical procedure. CONCLUSION;In the surgical managements of upper of upper tract TCC, laparoscopic nephroureterectomy dose not negatively affect long-term oncological control and can be an alternative modality. Tumor stage and grade are, however,important prognostic factors in the metastases and cancer specific mortality.

\section{WS1-2-4}

\section{Phase I Clinical Study of Anti-apoptosis Protein, Survivin-derived Peptide Vaccine Therapy for Patients with Advanced or Recurrent Urothelial Cancer}

Department of Urology, Sapporo Medical University, School of Medicine, Sapporo, Japan ${ }^{1)}$, 1st Department of Pathology, Sapporo Medical University, School of Medicine, Sapporo, Japan ${ }^{2}$, Department of Urology, Hakodate Goryokaku Hospital, Hakodate, Japan ${ }^{3)}$, Department of Urology, Oji General Hospital, Tomakomai, Japan ${ }^{4)}$

Ichiya Honma $^{1)}$, Hiroshi Kitamura ${ }^{12)}$, Toshihiko Torigoe ${ }^{2)}$, Yoshihiko Hirohashi ${ }^{2)}$, Eiji Sato ${ }^{12)}$, Atsushi Takahashi ${ }^{3)}$, Keisuke Taguchi' ${ }^{4}$, Naoya Masumori ${ }^{1)}$, Naoki Itoh $^{1)}$, Noriyuki Sato ${ }^{2)}$, Taiji Tsukamoto ${ }^{1)}$

[OBJECTIVE]: Survivin, a member of the inhibitor of apoptosis protein family, is expressed in various cancers such as colorectal, breast and urothelial cancers but is undetectable in normal adult tissues. Because of its cancer-specific expression, survivin is an ideal target for cytotoxic $\mathrm{T}$ lymphocyte (CTL)-based immunotherapy. Previously we reported that we established a survivin-2B peptide carrying the HLA-A24 binding motif and that CD8+ CTLs were successfully induced by stimulation with this peptide in vitro. On the basis of these observations, we have started a phase I clinical study of survivin-2B peptide vaccination for patients with advanced or recurrent urothelial cancer (UC) to evaluate its feasibility, safety, and clinical efficacy. [METHODS]: Eligible patients were those (i) had histologically confirmed UC expressing survivin and HLA class I, (ii) were HLA-A24 positive, (iii) between 20 and 80 years old, (iv) had ECOG performance status between 0 and 3 , and (v) who gave informed consent. Vaccinations with survivin-2B peptide vaccine with IFA serving as a carrier for a water-in-oil emulsion were given subcutaneously six times at 14-day intervals. These patients were evaluated for adverse events, took a DTH skin test, received tetramer analysis for evaluation of immunological responses, and their clinical responses were determined by imaging studies. [RESULTS]: A total of 36 vaccinations were carried out in 7 patients. No severe adverse events were observed in any patient. Slight reduction of the tumor volume was observed in one patient, who was considered a minor responder. Analysis of peripheral blood lymphocytes of this patient using HLA-A24/peptide tetramers revealed a significant increase in peptide-specific CTL frequency after 6 vaccinations. [Conclusions]: This study indicates that survivin-2B peptide vaccination is safe and should be further considered for potential immune and clinical efficacy in HLA-A24 positive survivin-expressing UC patients. 